\title{
An Influence of Positive Affect on Decision Making in Complex Situations: Theoretical Issues With Practical Implications
}

\author{
Alice M. Isen \\ Johnson Graduate School of Management and Department of Psychology \\ Cornell University
}

\begin{abstract}
This article reviews evidence indicating that, in most circumstances, positive affect enhances problem solving and decision making, leading to cognitive processing that is not only flexible, innovative, and creative, but also thorough and efficient. These results have implications regarding consumers' potential reactions to affect generated by ads, products, consumption situations, and service encounters.

These cognitive effects of positive affect are considered in the context of effects on social interaction that show that positive affect leads to helping, generosity, and interpersonal understanding. Together, these findings suggest implications especially for customer satisfaction, and particularly for a role for employee positive affect, or employee satisfaction, in generating customer satisfaction.

Moreover, studies specifically in the domain of medical decision making and problem solving indicate that these implications would apply to the specific areas of doctor-patient interaction, medical decision making, and medical consumer satisfaction. Finally, it is suggested that the benefits of positive affect be considered when formulating healthcare policy and be included in economic models and policy decisions more generally as well.
\end{abstract}

In recent years, there has been growing research interest, in both theoretical and applied fields, in the influence of affect, or feelings, on decision making and problem solving. It is now widely accepted that affect regularly plays a role in cognitive processes, and even mild positive affect is recognized today as an important influence on thinking (e.g., Isen, 1993b). There has been some debate over whether this influence is disruptive or facilitative, but now, on balance, it seems possible to resolve some of the uncertainty surrounding that question. This article summarizes some of the evidence suggesting that, as long as the situation is one that is either interesting or important to the decision maker, positive affect facilitates systematic, careful, cognitive processing, tending to make it both more efficient and more thorough, as well as more flexible and innovative. Second, in the course of presenting these ideas, this article very briefly considers their intersection with the large body of work on attitude toward the ad and brand, which actually involves issues related to affect, such as affect transfer and cognitive mediation of affect.

Requests for reprints should be sent to Alice M. Isen, Johnson Graduate School of Management, Cornell University, 359 Sage Hall, Ithaca, NY 14853-6201. E-mail: ami4@ cornell.edu
Third, this article considers some implications of these findings for customer satisfaction, both directly and as it may be influenced by employee satisfaction or employee positive affect. This is especially relevant for service industries, although it may also play an important role in other sectors as well. The extension of the work on positive affect to customer satisfaction specifically is a relatively new potential application that has not seen much research as yet, but it holds great potential for expanding understanding of customer loyalty, relationship marketing, and many other aspects of customer satisfaction. Integration of the role of employee satisfaction in generating and maintaining customer satisfaction is another potentially very important application of the affect work.

Another focus is applying these considerations in particular to medical consumers (previously known as patients). Because some of our recent work has been carried out in medical settings, this provides the opportunity to begin addressing, in particular, factors that influence medical consumer decision making and medical consumer satisfaction. This focus allows us to consider implications of the work on affect and decision making for understanding the influence of affect on doctor-patient interaction and other determinants of patient satisfaction. 
With the aging of the large "Baby Boom" generation, our country is anticipating a marked increase in the use of medical care. This, together with the change in the way medical care is administered, which has led to its being seen more as a business, has spawned an unprecedented interest in medical consumer satisfaction among healthcare organizations and practitioners. Furthermore, the relationships among medical care providers, consumers, payors, and companies are in a state of flux as well. Thus, this seems an opportune time to focus attention on questions related to determinants of patient satisfaction and to investigate some of the marketing implications of issues related to doctor-patient interaction, patient satisfaction, and the medical context.

Finally, another focus for this article arises from the fact that both patients and the public may be viewed as medical consumers. Consequently, our consideration of affect's influence on doctor-patient interaction, physician satisfaction, medical decision making, and medical consumer satisfaction may also be broadened to include matters relating to public healthcare policy and public policy more generally. That is, it may be timely to suggest that policy makers consider what is known about the influence of positive affect on cognition and social responsibility - in particular, how valuable positive affect is to society-when they make public policy decisions.

In the short space available in this article, I cannot consider all of these topics in depth, but it is my hope that this article serves to stimulate further research and consideration of these issues, and particularly of the relevance of positive affect for these issues among consumer researchers.

\section{SOME RELEVANT FINDINGS}

To understand the potential role of positive affect in consumer responses, customer satisfaction, employee satisfaction, doctor-patient interaction, and satisfaction with medical care, as well as potential policy implications, it is helpful to consider several sets of research findings that indicate some of the major influences that positive affect has been found to have on thinking and interpersonal interaction.

\section{Helpfulness and Generosity (Altruism)}

First, dozens of studies, over the past 30 years, showed that positive feelings promote helping and generosity, as measured by such things as donating to charity, helping someone pick up dropped papers, volunteering to serve as a helpful confederate (but not as a harmful confederate) in an experiment, making a call for someone who ostensibly used her last change in reaching a wrong number (you) on the telephone, and so forth (e.g., see Isen, 1987, for more detailed consideration).

\section{Creative Problem Solving}

Second, many studies found, using a variety of affect inductions and a variety of ways of measuring creativity and creative problem solving, that positive affect facilitates creativity, cognitive flexibility, innovative responding, and openness to information. People in whom positive affect is induced have been found to give unusual (but reasonable) first associates, and have a more diverse set of associates, to neutral words (Isen, Johnson, Mertz, \& Robinson, 1985); to categorize material, including categories of people, more flexibly (e.g., Dovidio, Gaertner, Isen, \& Lowrance, 1995; Isen \& Daubman, 1984; Isen, Niedenthal, \& Cantor, 1992; Murray, Sujan, Hirt, \& Sujan, 1990); to seek variety among safe, enjoyable products and have a broadened consideration set in choosing such products (Kahn \& Isen, 1993); to produce artistic creations that are judged as more creative (e.g., Hirt, Melton, McDonald, \& Harackiewicz, 1996); and to excel at solving problems usually thought to require ingenuity such as Duncker's (1945) Candle Task (Greene \& Noice, 1988; Isen, Daubman, \& Nowicki, 1987; see Isen, $1999 \mathrm{~b}$, for fuller discussion of this topic). Positive affect has even been found to increase people's scores on a subset of items from the Remote Associates Test (RAT; e.g., Isen et al., 1987). The RAT is an assessment instrument developed in accord with Mednick's (1962) theory of creativity and validated as an individual-difference measure of creativity (Mednick, Mednick, \& Mednick, 1964). This finding, that positive affect improves performance on the RAT, has been so robust that we now sometimes use performance on a subset of the test as a check on our induction of affect (e.g., Estrada, Isen, \& Young, 1994).

It should be mentioned that these effects of positive affect on problem solving, flexibility, and innovation were observed in a wide variety of applied settings and among diverse populations. For example, this effect of happy feelings on flexible thinking was studied in the literature on consumer decision making, extending to the way consumers think about and decide to purchase and use products and services (e.g., Barone, Miniard, \& Romeo, 2000; Isen, 1993a, 1993b; Kahn \& Isen, 1993; Lee \& Sternthal, 1999; Lewinsohn \& Mano, 1993; Mano, 1997). For example, positive affect was found to influence people's acceptance of brand extensions to product types that are less typical of a brand's usual domain: to influence their variety seeking in product choice, the size and range of the consideration set for a choice (i.e., the kind of items that are even considered), and the way products are categorized and thought about, just to name a few. It has also been observed in organizational settings and has been shown to influence managerial decision making, conflict resolution, team behavior, job task perception, and a host of other processes relevant to the life of organizations (e.g., George \& Brief, 1996; Isen \& Baron, 1991; Kraiger, Billings, \& Isen, 1989; Staw \& Barsade, 1993; Weiss, Nicholas, \& Daus, 1999). 
In addition, research into the processes of health psychology and coping with stressful life events also indicates that positive affect can facilitate coping processes and health-promoting behavior (e.g., Aspinwall, 1998; Aspinwall \& Taylor, 1997; Taylor \& Aspinwall, 1996). People in whom positive affect has been induced tend, more than controls, to take a problem-solving approach to interpersonal problems and disputes, and come up with the kind of solution that involves thinking creatively about how to obtain the most for both sides. There is even indication that they are better able to take the other party's perspective in negotiation and are less "defensive" (Carnevale \& Isen, 1986). They also appear less defensive in stressful situations and seem to engage less in defensive interpersonal processes such as downward comparison, competitive comparisons, making oneself feel better by focusing on the relatively worse outcome of another, or feeling threatened by a friend's success (e.g., Aspinwall, 1997, 1998; Trope \& Neter, 1994; Trope \& Pomerantz, 1998).

It may be appropriate here to point out that the effects discussed in this article are different from, and supplement, most of those in the attitude-toward-the-ad literature (see e.g., Brown, Homer, \& Inman, 1998). That is, in addition to direct effects of affect such as affect transfer, and beyond effects that might be called "indirect" effects, involving cognitive processes such as interpretations and thoughts influenced by positive affect (e.g., Isen, 1987, 1993a, 1999a; Isen, Shalker, Clark, \& Karp, 1978; Kim, Allen, \& Kardes, 1996; Schwarz $\&$ Bless, 1991), the work discussed here indicates that there are also effects of positive feelings on cognitive organization and processes that result from changes in cognitive organization. For example, growing evidence indicates that positive affect influences how material is categorized and thought about, what is seen as related to what, and so forth (e.g., Barone et al., 2000; Isen, 2000; Isen \& Daubman, 1984; Isen et al., 1992; Lee \& Sternthal, 1999).

In fact, some articles in the attitude-toward-the-ad literature also found results compatible with those reported here that suggest that effects on thoughts and inferences, not just direct affect transfer, occur in response to affective interventions, as, for example, Kim et al. (1996). For another example, Brown et al. (1998), in their meta-analysis, reported findings that appear to support what they call a "contingent asymmetry" relation between positive and negative affect and setting conditions. That is, they concluded that positive and negative affect interact differently with various setting conditions, such as experimental instructions and cover story, in impacting attitudes. This suggests in another way that affect does not just transfer automatically, but that cognitive processes involving people's goals and understandings play a role. These results may also support the argument that positive and negative affect are not polar opposites (or at least not functionally so), but instead may be separate constructs-an idea suggested earlier (e.g., Isen, 1984; Watson, Clark, \& Tellegen, 1988; Watson \& Tellegen, 1985), but now under debate again (e.g., Russell \& Carroll, 1999a, 1999b; Watson \& Tellegen, 1999).
But this article includes evidence that addresses these more complex cognitive processing changes in more detail. For example, Brown et al. (1998), in introducing and considering their meta-analysis, argued (following, e.g., Bless, Bohner, Schwarz, \& Strack, 1990; Schwarz, 1990) that negative affect would lead to more elaboration than positive, but also argued (following, e.g., Mackie \& Worth, 1991) that positive affect cues a lot of material, which depletes cognitive capacity. In contrast, what the literature in the field now shows is that increased thinking, cognitive elaboration, is characteristic of positive affect, and that it does not, in fact, lead to depletion in cognitive capacity or depletion in motivation to process systematically (e.g., Isen, 1987, 1993b, 2000; Isen et al., 1985; Isen, Rosenzweig, \& Young, 1991; Isen et al., 1978; Kahn \& Isen, 1993; Lee \& Sternthal, 1999). Recently Schwarz and his colleagues have acknowledged this point and presented evidence supporting it (e.g., Bless et al., 1996). But, beyond the recognition that positive affect does not interfere with systematic processing, the realization that it promotes elaboration and innovative thinking means that marketers must anticipate and work with the deeper, richer thinking that will result from positive affect.

Thus, the work to be summarized in this article indicates that, in addition to simple effects, positive affect changes cognitive processing in major ways, through effects such as elaboration and increased thinking about a wide range of related ideas, and that this increased elaboration facilitates cognitive organization and capacity rather than depleting it. One implication of these findings is that aspects of ads or consumption environments that induce positive affect, such as humor, for example, because they increase thinking (about interesting things) and cognitive flexibility, may lead consumers to think about many different brands, or related products, not just the advertised one. Thus, these details must be understood and planned for in utilizing positive affect.

\section{Variety Seeking}

Third, a series of studies indicated that positive affect promotes variety seeking among safe, enjoyable consumer food products as measured by number of switches and market share of the most preferred brand (Kahn \& Isen, 1993). It was also found that the consideration set was larger among people in whom positive affect had been induced, and, as indicated earlier, in the context of creativity or flexibility in thinking, the range of items included in a consumption category (e.g., soup, snacks) was larger among positive-affect subjects, and extended to nontypical category items.

It should be noted that results relating to variety seeking should not be taken to imply that people in positive affect will be likely to take dangerous risks. First, in the variety-seeking studies themselves, results indicated that these effects held only where the products were safe and enjoyable. Second, there are data on affect and risk taking that, 
similarly, show that positive affect does not promote real risk taking: In several studies, findings consistently indicated that people in whom positive affect was induced, compared to controls, avoided a real, meaningful risk (e.g., Arkes, Herren, \& Isen, 1988; Isen \& Geva, 1987; Isen \& Patrick, 1983). In addition, other studies indicated that these results may reflect a greater negative utility for a loss among people who are feeling happy (Isen, Nygren, \& Ashby, 1988; Nygren, Isen, Taylor, \& Dulin, 1996).

\section{Negotiation}

Another relevant domain involves negotiation processes. In a study of integrative bargaining, positive affect was found to facilitate the bargaining process and to improve the outcomes of face-to-face negotiators trying to reach agreement on buying and selling appliances (Carnevale \& Isen, 1986). In this study, people negotiating face-to-face obtained better outcomes in the negotiation and enjoyed the process more, when positive affect had been induced. In the control condition, not only was the mean joint outcome for the negotiators significantly lower than that obtained in the affect condition, but the modal response was to break off negotiation and not reach any agreement at all. In contrast, those in the affect condition were most likely to reach the optimal agreement possible for each party. In the control condition, face-to-face bargainers became quite hostile and the situation deteriorated into a contentious, angry one; but in the positive-affect condition, people were much less likely to engage in aggressive tactics during the negotiation and reported enjoying the session. They took a problem-solving approach and were also better able to see the situation from the other person's perspective (as reflected by the finding that they were better able to report the other negotiator's payoff schedule, a fact not divulged during the negotiation). Thus, there is reason to believe that, even in a potentially hostile situation, positive affect facilitates cognitive flexibility, the ability to switch perspectives and see things in multiple ways and come up with viable solutions, and the ability to cope with potential problems and avoid conflict.

\section{Decision Making}

The findings of our decision-making studies are very useful in this context, also. This work suggests that, as long as the task is one that is meaningful, interesting, or important to the decision maker, positive affect leads to decision making that is both more efficient and more thorough. For example, in a study involving choice among six hypothetical cars for purchase (based on information from nine dimensions), people in whom positive affect had been induced were more efficient in the way they went about the complex task (Isen \& Means, 1983). That is, although their ultimate choices did not differ from those of control participants, they reached a decision sooner, showed less redundancy in the search process, and eliminated two dimensions from consideration, but only dimensions that were of low importance.

When this same paradigm, modified to simulate medical diagnosis, was used to study the medical decision-making processes of young medical-care providers in training (4th-year medical students with 1 full year of clinical experience), results compatible with those just described were obtained. Clinicians in whom positive affect had been induced (by means of report of success on an anagram task) were more efficient in completing the assigned task, which was to decide which of six hypothetical patients was most likely to have lung cancer, based on information from nine different dimensions (Isen et al., 1991). In this study, however, where the task was more substantive than choice of a hypothetical car for purchase, protocol analyses revealed three additional effects. First, people in the positive-affect condition were significantly more likely to go beyond the assigned task and do more than was required; second, they showed significantly less confusion in their decision process; and third, they showed significantly more integration of information for decision making.

As noted, protocol analyses showed that positive-affect participants were significantly more likely than controls to do more than was asked-to go beyond the task. Primarily what they did, to a significantly greater extent than control participants, was to attempt to diagnose the remaining cases, and, in some instances, they suggested treatments. Thus, they were more thorough than controls in their consideration of the materials.

The findings of the car-choice and medical-diagnosis studies suggest that, under conditions of positive affect, people tend to integrate material for decision making and be less confused by a large set of propositions. This allows them to work faster and either finish earlier (as in the case of the car-choice task) or to turn attention to other important or interesting tasks within the materials (as in the medical-diagnosis task).

Two follow-up studies looked at the influence of positive affect on the diagnostic processes of practicing physicians, ranging in age from 30 to 70 , in a hospital setting (Estrada, Isen, \& Young, 1994; Estrada, Isen, \& Young, 1997). Participants were asked to read a brief description of a patient and to think aloud, as they tried to reach a diagnosis. As in the earlier decision-making studies described, each participant was tested alone, and his or her responses were tape-recorded and later transcribed for analysis. Participants were presented with folders containing information regarding the patient's history, physical exam, laboratory tests, and so forth, corresponding to the categories used by physicians in making a diagnosis. Every piece of information was covered with a paper tab, and the physician-participants could uncover whatever information they felt they needed to make the diagnosis. Those who had been randomly chosen to be in the positive-affect condition received a small bag of wrapped candy, similar to that used in previous research, but containing six hard can- 
dies and four Hershey's Miniatures chocolates. This was given to them in a large envelope that also contained the folders and other materials for the study, and they were asked, via written instructions, to put the candy away and not to eat any right then, so that the experimenter would not know their experimental condition while he interacted with them.

Before beginning the diagnostic task itself, participants were asked to take the seven-item RAT described earlier in discussing the influence of positive affect on creativity. In this instance, performance on the RAT was intended as a check on the manipulation of affect. We reasoned that if the positive-affect doctors performed better on the RAT, we would have indication that our positive-affect manipulation had been effective. In addition, we would know that doctors in whom positive affect had been induced as in previous experiments also showed increased creative problem-solving ability, a skill which may actually aid in diagnosis, because medical diagnosis requires seeing connections among elements of a case or situation.

Results of these studies showed several important effects: First, doctors in whom positive affect had been induced by receiving a small package of candy showed significantly better performance on the RAT of creative problem solving (Estrada et al., 1994). Second, in solving the diagnostic problem, for which the correct diagnosis was chronic active hepatitis, doctors in the positive-affect condition realized that the symptoms might suggest liver disease, and considered liver, significantly earlier in their protocols than control participants. They did not "close" on this category sooner than controls, but they did begin consideration of it earlier in their decision process. In other words, they more readily put together the signs and symptoms, to realize that liver disease was a possibility (correct hypothesis generation). Several other measures indicated that this was not due to premature closure, "closed thinking," or other such undesirable effect that might indicate "sloppy" processing, jumping to a conclusion, or heuristic processing, rather than efficient, effective, careful processing. For example, they considered just as many diagnoses as control participants before, and after, the liver domain was first recognized as the likely domain of the illness (Estrada et al., 1997).

Second, the results indicated that positive affect significantly reduced the tendency to engage in anchoring in the diagnostic-reasoning process. Anchoring is related to the Bayesian concept of estimating and adjusting the probability of an event in light of additional evidence, and as used in this study, it connotes inadequate adjustment to new information, specifically here the tendency to hold onto an incorrect hypothesis by distorting or ignoring disconfirming evidence. As with the other measures in the protocol analysis, this measure was assessed by two independent raters, not aware of the experimental condition of the protocol when scoring it. Results of this analysis indicated that physicians in the positive-affect condition showed significantly less anchoring than controls (Estrada et al., 1997).
As an aside, it should be mentioned that this finding may imply that positive affect may reduce the magnitude of a wide range of similar phenomena such as assimilation, primacy, stereotyping, correspondence bias, the perseverance effect, overconfidence, and possibly the illusory truth effect as discussed by, for example, Hawkins and Hoch (1992). But, again, as has been discussed, this influence of positive affect may interact with the materials being considered and may apply only to interesting or important (in the marketing context, "involving") materials, products, and decision situations. Also, to say that positive affect may reduce the magnitude of these effects does not mean that it will make the primed material, the stereotype components, or the material seen first (the primacy effect) less accessible, but rather that it will reduce the difference in accessibility, or utilization, between such material and other material. In other words, positive affect may increase accessibility of schemata such as stereotypes, but also increase or leave relatively high the accessibility of alternatives and the ability to hold and use both in mind simultaneously, or to switch between/among them in thinking (see Ashby, Isen, \& Turken, 1999; Isen, 1999a, for discussions of the possible neuropsychological basis for such improved switching ability as a result of positive affect).

Indeed, Lee, and Sternthal (1999) recently reported something like a reduction in primacy effects, in the sense of less of a serial-position effect-not impaired recall of items early in the list, but improved recall for middle positions in serially presented items - among people in whom positive affect had been induced, compared with controls. In addition, although some studies have investigated positive affect's influence on, for example, stereotyping, and have reported that it increases it, those results appear to be dependent on the nature of the materials or task situation, or both. In one set of studies, for example, such effects disappeared when the importance of the task was increased (participants were made accountable for their answers; Bodenhausen, Kramer, \& Susser, 1994). This latter finding indicates, as does other work (see, e.g., Isen, 2000, for discussion), that details related to factors such as involvement, nature of the task materials, and so forth, will likely interact with affect in determining these effects. Clearly, further research is needed to investigate many of these interesting possibilities.

Finally, returning to discussion of the study on physician diagnostic processes, after solving the diagnostic problem, the physicians filled out a questionnaire indicating the perceived importance, for them, of two different types of sources of their own satisfaction from the practice of medicine-humanistic and extrinsic. An example of a humanistic item, taken from tests of "humanism in medicine" (e.g., Abbott, 1983; Butterfield \& Mazzaferri, 1991; Hauck, Zyzanski, Alemagno, \& Medalie, 1990) is "By establishing rapport with patients, I enhance my human understanding." An example of an extrinsic item is "Yearly income is very important for practice satisfaction." Results indicated both a main effect of type of motivation and an interaction between affect and type of 
motivation: Doctors, in general, gave higher importance ratings to the humanistic sources of satisfaction from practicing medicine; however, there was also a significant interaction between affect and item type, indicating that this difference in favor of the humanistic items was even more pronounced for the positive-affect doctors (Estrada et al., 1994). Thus, even though this task took place about $40 \mathrm{~min}$, and two intervening tasks, after the mild affect induction, results revealed a significant difference between the affect and control conditions on this task designed to investigate physicians' motivation in medicine or orientation to patients.

To summarize the work reported thus far, it appears that positive affect generally leads people to be gracious, generous, and kind to others; to be socially responsible; and to take the other's perspective better in interaction (while not losing sight of their own perspective). Decision makers and problem solvers in whom positive affect has been induced are more flexible, open, and innovative, as well as more careful and thorough in addressing interesting or important issues. There is no evidence that they suffer from reduction in cognitive capacity, or that their motivation to process systematically is disrupted. To the contrary, they seem, if anything, more motivated, more thoughtful, better organized, and more clear-headed.

The work adding medical practitioners to this body of research suggests that positive affect sufficient to influence cognitive processes and promote a more caring attitude toward others can be induced as readily in physicians in their professional surroundings as it can be in other populations who have been studied. This work also suggests that such positive affect can lead doctors to improved diagnostic processes, as well as to a more humanistic or patient-focused approach to medicine. In other words, doctors in whom positive affect has been induced may be more understanding, in both senses of the word (that is, better able to see connections in material and thus solve diagnostic problems, and more understanding of their patients as people). This would be completely in keeping with the influence of positive affect that has been found in nonmedical settings over the past 25 years: It tends to promote kindness, helpfulness, and taking the other person's perspective, as well as improved performance on tasks requiring complex problem-solving skills (on both interpersonal and nonsocial tasks).

In addition, these results suggest several important, general points about positive affect's influence on cognitive processing: First, that positive affect can lead to more thorough and careful problem solving and decision making, at the same time that it leads to efficiency in considering the materials. Thus, even though people in positive affect may be more efficient or quicker in deciding, it is probably not most informative to think of positive affect as disrupting systematic processing and leading to faulty, hurried, or incomplete, sloppy decision making, as was argued for a time (e.g., Mackie \& Worth, 1989; Schwarz \& Bless, 1991). Positive affect appears to facilitate systematic, careful processing and need not disrupt systematic processing or, by its nature, reduce cognitive capacity (see Isen, 1993b, 1999a, 2000, for discussion of this issue, and Bless et al., 1996, for a retraction of their earlier view that positive affect interferes with the motivation to process systematically).

The second point suggested by these results, taken together, is that the impact of positive affect will depend on the nature of the materials and the task assigned, in part because it depends primarily on what the person is trying to do or wants to do in the situation, and in part because the materials play a role in what the person thinks about. In fact, it may be that earlier findings that seemed to suggest that positive affect impaired careful processing (e.g., Mackie \& Worth, 1989; Melton, 1995; Schwarz \& Bless, 1991) could have been a function of the particular materials and task situations involved. An ongoing study is currently investigating this possibility (Isen, Christianson, \& Labroo, 2001).

A third point that might also be mentioned is that specific measures (like total time working on the task materials) that are appropriate in one situation or with one set of materials (as was found, for example, in the car-choice study described previously; Isen \& Means, 1983) may not be valid in another situation or with different materials (as was found with the medical-choice study; Isen et al., 1991), and therefore should not unquestioningly or automatically be applied or interpreted. That is, in those two studies, the same basic processes were observed, but the measures that reflected those processes were different because of the different materials or tasks.

The point that the impact of affect depends on consumers' thinking processes, and not simply automatic processes, also broadens our understanding of both affect and the nature of its impact on consumer thinking. As noted earlier, this is reflected in the debates within the attitude-toward-the-ad literature (e.g., Brown et al., 1998; Kim et al., 1996). But in addition, advertising or other aspects of marketing that involve use of positive affect will need to consider that positive affect, because of the flexibility, elaboration, and thinking it introduces, may completely change people's ways of thinking. Thus, humor in ads, for example, may lead people to think about other brands or even other products or alternative ways to spend their money to accomplish their goals. And such possibilities need to be considered when planning marketing strategy in a positive-affect context.

Furthermore, the important effects of materials and context, which have been observed repeatedly, suggest that factors such as involvement may also be important to consider in anticipating the impact of positive affect and working with it. One implication of this observation is that the effects may be more likely to be observed with important or interesting choices, for example, than with unimportant or boring decisions. However, the form of "involvement" that may be most important may be interestingness of the materials or choices. That is, even "unimporant" choices may show the effects of increased thinking or cognitive reorganization if they are in- 
teresting, charming, or enjoyable to think about. This is another topic for further research.

\section{IMPLICATIONS FOR CUSTOMER SATISFACTION}

All of these findings can be applied to consumer contexts of both decision making and customer satisfaction, including medical consumer (patient) decision making and satisfaction. The implications of these findings for consumer decision making are being explored in many contexts, including the informative work on both direct and indirect effects on attitude-toward-the-ad (e.g., Brown et al., 1998; Kim et al., 1996), as well as other work, at the next level of complexity and integration, on positive affect's influence on consumer decision making (e.g., Barone et al., 2000; Kahn \& Isen, 1993; Lee \& Sternthal, 1999).

However, less well-recognized are the implications for customer satisfaction, and especially the potential role of employee satisfaction in generating and maintaining customer satisfaction. (See Rucci, Kim, \& Quinn, 1998; Schneider, Ashworth, Higgs, \& Carr, 1996; Schneider \& Bowen, 1985; Schneider, White, \& Paul, 1998, for discussion of the link between employee attitudes, and possibly satisfaction, and customer satisfaction from a management or organizational behavior perspective.) As work in the management literature is showing-for example, the recent study conducted at Sears, Roebuck, \& Co. (Rucci et al., 1998)—employee attitudes, and possibly satisfaction, can lead to customer satisfaction. What the work presented in this article suggests is that positive affect in employees may play a role in this process.

For example, as data reviewed in this article show, positive affect promotes a helpful and generous orientation to others, as well as an enhanced problem-solving capability and a more open and flexible decision-making and problem-solving style. Thus, it is not unreasonable to propose that, in interacting with customers, employees in whom positive affect has been induced may be more flexible, understanding, and helpful, as well as better able to address the customer's task or concern. In addition, helpfulness and liking are usually reciprocated (e.g., Berkowitz \& Daniels, 1964; Gouldner, 1960). Consequently, this body of research on positive affect suggests, in general, that inducing positive affect in staff members who interact with the public may result in improved customer response, satisfaction, and loyalty. Thus, this work points to an intersection between areas traditionally seen as within the marketing function (customer satisfaction) of the organization and ones usually considered in the realm of organizational behavior, human resources, or management (employee satisfaction and productivity).

Applying this realization in a medical context seems especially important and potentially of far-reaching impact. And given that several of the relevant effects of feelings on social interaction and understanding were obtained in a medical context, using medical practitioners as participants, it seems a promising possibility.

However, the task is not completely simple. A recent article highlighted some of the differences or complexities that arise when one attempts to address marketing issues or concepts in a medical context (Kahn et al., 1997). This is true for both decision making and customer satisfaction. Concepts such as involvement, stress, risk, assessment of satisfaction, pricing, and promotion, among others, all have unique dimensions in a medical context.

For example, in the medical context, unlike most other consumer contexts, the very question of whether patients should participate in the decision-making process, and if so, to what extent, is a controversial one. This does not refer to patients' participating in the diagnostic process, but rather to their participating in other medically related decisions having to do with treatment alternatives, where patient preferences, values, and so forth, may be relevant. Even here, the degree of patient participation in medical decisions, or the balance between doctor and patient in who is responsible for making decisions, is an issue (e.g., Kassirer, 1994; O'Connor et al., 1999). There are various factors that play a role in these patient preferences, including the patient's age, education level, the type of medical condition, the doctor's own preference, and so forth. Further complicating the picture, patient participation in decision making has been found to influence patient satisfaction (e.g., Kuder, Isen, \& DeLia, 1996; Pontes \& Pontes, 1996). Thus, the stage is set for some unexpected relationships and factors influencing consumer (patient) satisfaction.

For example, as noted, research is beginning to show a link between patient participation in decision making and patient satisfaction with medical care. One recent study (Pontes \& Pontes, 1996) reported evidence suggesting that when doctors enable patients to participate in the medical decision process, patient inferences about the quality of care and about physician skill and concern are more favorable, as are patient satisfaction ratings. In another article, compatibly, it was found that patients who preferred more participation in the medical decision-making process also reported higher levels of satisfaction (Kuder et al., 1996).

Consequently, the data showing that physicians in whom positive affect has been induced take a more humanistic orientation to the practice of medicine and are more open and flexible in considering information may have important implications for the situation, because they suggest that positive affect may lead doctors to be more open to patient participation in the medical decision processes. That is, if the increased physician humanism that results from positive affect leads to increased sharing of decision-making involvement between physician and patient, there is reason to believe that this will have a positive influence on patient satisfaction.

A second important way in which positive affect may play a role in patient satisfaction is through its potential impact on 
the doctor-patient interaction. Both patient participation and patient satisfaction may be related to the quality of the doctor-patient interaction.

At first blush, this may seem self-evident and not in need of a boost from positive affect. It may seem that doctors would understand the importance of good-quality interaction with patients. However, in the medical sector even more than in others, determinants of patient satisfaction are very complex, sometimes depending predominantly on factors other than the doctor-patient interaction, such as medical outcome. In medicine, therefore, the doctor-patient interaction is often viewed as secondary to the main point of the medical interaction, the patient's recovery, at least among physicians. Thus, the doctor-patient interaction is often even seen as unimportant by physicians, and their interpersonal skills or nuances of their interactions with patients may not be of central concern to them. Consequently, in actual practice, physicians may lose sight of the importance of the quality of their interaction with patients and of the small things that they may say or do that actually impact patients significantly.

To illustrate this situation, consider that one complaint that is frequently heard about doctor-patient interactions is that the participants do not seem to understand each other-that is, most doctors feel that they "care" about their patients, but many patients feel that their doctors are not listening to them or do not understand when they do listen. Thus, it seems important to find ways to increase understanding in this context. For example, studies have now reported that, on average, doctors interrupt their patients and take control of the conversation after less than $20 \mathrm{sec}$ of the medical interview (e.g., Beckman \& Frankel, 1984). Similarly, with many doctors it is difficult for patients to participate in the decisions surrounding their own health care (e.g., O'Connor et al., 1999), and there is evidence that doctors and patients do not even agree on the relative amounts that they participated in a given decision. Thus, there appears to be quite a bit of misunderstanding in the doctor-patient service encounter.

The work discussed in this article suggests that perhaps one way of beginning to address this cycle of misunderstanding and potential resentment may be through facilitating positive affect in physicians. That is, perhaps encouraging doctors to feel competent and valued may help them not only to address the medical problem, but also to take a more humanistic, valuing approach to the patient. If they listen longer, hear the patient out, encourage, and support patient participation in the decision making, patients may feel valued and genuinely respected, which could then influence patient satisfaction and loyalty as patients reciprocate the positive regard.

Thus, the medical situation, especially, contains many possibilities for misunderstandings and many possibly missed opportunities for improving patient care and patient satisfaction that can be improved by positive affect in the providers of care. Given that positive affect influences people in general to be more helpful and kind, and may influence doctors to be more patient-centered in approach, positive affect may be an important tool for addressing these problems: It may promote the doctor's seeing the importance of the doctor-patient interaction, and it may increase his or her ability to be understanding and kind. It may lead the doctor to encourage, or at least be accepting of, appropriate patient participation in decision making, which itself may contribute to patient satisfaction. And finally, it may promote flexibility, not only in the way the healthcare provider interacts with the patient, but also in addressing and solving the medical problem.

\section{POLICY IMPLICATIONS}

It should be noted that this process-indeed anything that increases understanding between doctors and patients-may have implications for larger, societal approaches to healthcare, especially in this age of managed care. However, this influence may be in a direction counter to the current trend of looking at people as large aggregates, and looking at healthcare primarily in terms of dollars-and-cents economic issues. With more emphasis on humanistic concerns and on viewing patients as unique, capable, coping fellow human beings, doctors may refocus on the needs of their individual patients. Caring and understanding may lead to more valuing of each individual, a tendency that may not be compatible with rigid adherence to formulae for rationing of medical care based on broadly defined, aggregate characteristics such as age. Furthermore, increased patient participation in healthcare-related decisions may also place focus on individuals.

Nonetheless, if positive affect improves trust and honest communication between doctor and patient, perhaps more reasonable patterns of expenditures can still be achieved. If doctors do not need to practice defensive medicine, and patients can believe that their physicians have their best interests at heart, perhaps appropriately cost-effective courses of action may still be followed (e.g., Kuder, 1995). These are all topics on which research is needed at present.

Finally, this work showing an impact of positive affect on thinking and social behavior has implications for societal concerns, more broadly. In the domain of health care, as discussed, we appear in danger of losing sight of the individual and losing sight of the importance of some of the "implicit," or less obvious (nonmonetary), costs and benefits that need to be considered. Most economic models do not consider the beneficial effects that society obtains from the positive affect of its citizens-innovation, generosity, helpfulness, for example — or the costs that will be incurred (loss of these qualities) if we fail to maintain high morale.

For example, the research reviewed here would suggest that our society benefits, in ways not acknowledged by most economic accounts, from factors such as high morale and sense of self-worth, just to name two, that are common in our culture. Speculating, these morale factors may come, in part, from our society's high regard for each individual, and from 
the sense, which most people have, that society considers every life, including theirs-whether they are 75-years-old or 35 -years-old - as worth saving. An important point to consider, then, is that the costs involved in destroying such morale factors may be great; yet these costs and benefits are not usually considered in economic cost-benefit analyses.

Recently, however, an economic model has been proposed that specifically addresses the role of positive affect and illustrates the tangible value that results from maintaining positive affect and the potential costs of ignoring it (Hermalin \& Isen, 1999). Thus, there is growing support for the suggestion that it is important to consider, explicitly, the value of these implicit (nonmonetary) benefits, in formal economic analyses. Hopefully, in the near future this recognition of the importance and value of positive affect will also be applied to cost-benefit analyses in the establishment of public policy, including healthcare policy.

\section{CONCLUSION}

In conclusion, then, we have learned that positive affect, beyond its important effects on memory for, and attitudes toward, ads and brands, fosters clear-headed, well-organized, open-minded, flexible problem solving and thinking. These indirect results have many important implications for understanding consumer thinking, decision making, responses, and choices. Furthermore, these results, combined with the enhanced social skills and kindness that also result from positive affect, suggest that positive affect in employees may lead to more effective, as well as happier, employees - and this, in turn, may impact customer satisfaction. Thus, we have seen a potential avenue for integration of human resources and marketing concerns within companies. And we have considered how improving employee satisfaction can lead to improved customer satisfaction in one industry, healthcare, where improvement in employee-customer communication and understanding would be welcomed by most parties. Finally, we also considered the broader societal impact (benefit) of positive feelings, and the potential societal cost of losing these beneficial byproducts of happy feelings, and called for their recognition by policy makers.

\section{ACKNOWLEDGMENTS}

I thank Editor Frank Kardes for his many helpful comments and suggestions on this article.

\section{REFERENCES}

Abbott, L. C. (1983). A study of humanism in family physicians. Journal of Family Practice, 16, 1141-1146.
Arkes, H. R., Herren, L. T., \& Isen, A. M. (1988). Role of possible loss in the influence of positive affect on risk preference. Organizational Behavior and Human Decision Processes, 42, 181-193.

Ashby, F. G., Isen, A. M., \& Turken, A. U. (1999). A neuropsychologica 1 theory of positive affect and its influence on cognition. Psychological Review, 106, 529-550.

Aspinwall, L. G. (1997). Future-oriented aspects of social comparisons: A framework for studying health-related comparison activity. In B. P. Buunk \& F. X. Gibbons (Eds.), Health, coping, and well-being: Perspectives from social comparison theory (pp. 125-165). Mahwah, NJ: Lawrence Erlbaum Associates, Inc.

Aspinwall, L. G. (1998). Rethinking the role of positive affect and self-regulation. Motivation and Emotion, 23, 1-32.

Aspinwall, L. G., \& Taylor, S. E. (1997). A stitch in time: Self-regulation and proactive coping. Psychological Bulletin, 121, 417-436.

Barone, M. J., Miniard, P. W., \& Romeo, J. B. (2000). The influence of positive mood on brand extension evaluations. Journal of Consumer Research, 26, 387-402.

Beckman, A. B., \& Frankel, R. M. (1984). The effect of physician behavior on the collection of data. Annals of Internal Medicine, 101, 692-696.

Berkowitz, L., \& Daniels, L. R. (1964). Affecting the salience of the social responsibility norm: Effects of past help on the response to dependency relationships. Journal of Abnormal and Social Psychology, 68, 275-281.

Bless, H., Bohner, G., Schwarz, N., \& Strack, F. (1990). Mood and persuasion: A cognitive response analysis. Personality and Social Psychology Bulletin, 16, 331-345.

Bless, H., Schwarz, N., Clore, G. L., Golisano, V., Rabe, C., \& Wolk, M. (1996). Mood and the use of scripts: Does a happy mood really lead to mindlessness? Journal of Personality and Social Psychology, 71, 665-679.

Bodenhausen, G. V., Kramer, G. P., \& Susser, K. (1994). Happiness and stereotypic thinking in social judgment. Journal of Personality and Social Psychology, 66, 621-632.

Brown, S. P., Homer, P. M., \& Inman, J. J. (1998). A meta-analysis of relationships between ad-evoked feelings and advertising responses. Journal of Marketing Research, 35, 114-126.

Butterfield, P. S., \& Mazzaferri, E. L. (1991). A new rating form for use by nurses in assessing residents' humanistic behavior. Journal of General Internal Medicine, 6, 155-161.

Carnevale, P. J. D., \& Isen, A. M. (1986). The influence of positive affect and visual access on the discovery of integrative solutions in bilateral negotiation. Organizational Behavior and Human Decision Processes, 37, $1-13$.

Dovidio, J. F., Gaertner, S. L., Isen, A. M., \& Lowrance, R. (1995). Group representations and intergroup bias: Positive affect, similarity, and group size. Personality \& Social Psychology Bulletin, 21, 856-865.

Duncker, K. (1945). On problem solving. Psychological Monographs, 58 (Whole No. 5).

Estrada, C. A., Isen, A. M., \& Young, M. J. (1997). Positive affect facilitates integration of information and decreases anchoring in reasoning among physicians. Organizational and Human Decision Processes, 72, 117-135.

Estrada, C., Isen, A. M., \& Young, M. J. (1994). Positive affect influences creative problem solving and reported source of practice satisfaction in physicians. Motivation and Emotion, 18, 285-299.

George, J. M., \& Brief, A. P. (1996). Motivational agendas in the workplace: The effects of feelings on focus of attention and work motivation. In L. L. Cummings \& B. M. Staw (Eds.), Research in organizational behavior (Vol. 18, pp. 75-109). Greenwich, CT: JAI.

Gouldner, A. W. (1960). The norm of reciprocity: A preliminary statement. American Sociological Review, 25, 161-178.

Greene, T. R., \& Noice, H. (1988). Influence of positive affect upon creative thinking and problem solving in children. Psychological Reports, 63, 895-898. 
Hauck, F. R., Zyzanski, S. J., Alemagno, S. A., \& Medalie, J. H. (1990). Patient perceptions of humanism in physicians: Effect on positive health behaviors. Family Medicine, 22, 447-452.

Hawkins, S., \& Hoch, S. (1992). Low-involvement learning: Memory without evaluation. Journal of Consumer Research, 19, 212-225.

Hermalin, B., \& Isen, A. M. (1999). The effect of affect on economic and strategic decision making. Unpublished manuscript, Cornell University, Ithaca, NY.

Hirt, E. R., Melton, R. J., McDonald, H. E., \& Harackiewicz, J. M. (1996). Processing goals, task interest, and the mood-performance relationship: A mediational analysis. Journal of Personality and Social Psychology, 71, 245-261.

Isen, A. M. (1984). Toward understanding the role of affect in cognition. In R. Wyer \& T. Srull (Eds.), Handbook of social cognition (pp. 179-236). Hillsdale, NJ: Lawrence Erlbaum Associates, Inc.

Isen, A. M. (1987). Positive affect, cognitive processes, and social behavior. In L. Berkowitz (Ed.), Advances in experimental social psychology (Vol. 20, pp. 203-253). New York: Academic.

Isen, A. M. (1993a). The influence of positive affect on cognitive organization: Some implications for consumer decision making in response to advertising. In A. Mitchell (Ed.), Advertising exposure, memory, and choice (pp. 239-258). Hillsdale, NJ: Lawrence Erlbaum Associates, Inc.

Isen, A. M. (1993b). Positive affect and decision making. In M. Lewis \& J. Haviland (Eds.), Handbook of emotion (pp. 261-277). New York: Guilford.

Isen, A. M. (1999a). Positive affect. In T. Dalgleish \& M. Power (Eds.), The handbook of cognition and emotion (pp. 521-539). Sussex, England: Wiley.

Isen, A. M. (1999b). Positive affect and creativity. In S. Russ (Ed.), Affect, creative experience, and psychologica ladjustment (pp. 3-17). Philadelphia: Bruner/Mazel.

Isen, A. M. (2000). Positive affect and decision making. In M. Lewis \& J. Haviland-Jones (Eds.), Handbook of emotions (2nd ed., pp. 417-435). New York: Guilford.

Isen, A. M., \& Baron, R. A. (1991). Positive affect in organizations. In L. Cummings \& B. Staw (Eds.), Research in organizational behavior (pp. 1-52). Greenwich, CT: JAI.

Isen, A. M., Christianson, M., \& Labroo, A. A. (2001). A role for the nature of the task in determining whether positive affect facilitates performance. Unpublished manuscript, Cornell University, Ithaca, NY.

Isen, A. M., \& Daubman, K. A. (1984). The influence of affect on categorization. Journal of Personality and Social Psychology, 47, 1206-1217.

Isen, A. M., Daubman, K. A., \& Nowicki, G. P. (1987). Positive affect facilitates creative problem solving. Journal of Personality and Social Psychology, 52, 1122-1131.

Isen, A. M., \& Geva, N. (1987). The influence of positive affect on acceptable level of risk: The person with a large canoe has a large worry. Organizational Behavior and Human Decision Processes, 39, 145-154.

Isen, A. M., Johnson, M. M. S., Mertz, E., \& Robinson, G. F. (1985). The influence of positive affect on the unusualnes s of word associations. Journal of Personality and Social Psychology, 48, 1413-1426.

Isen, A. M., \& Means, B. (1983). The influence of positive affect on decision-making strategy. Social Cognition, 2, 18-31.

Isen, A. M., Niedenthal, P., \& Cantor, N. (1992). The influence of positive affect on social categorization. Motivation and Emotion, 16, 65-78.

Isen, A. M., Nygren, T. E., \& Ashby, F. G. (1988). The influence of positive affect on the perceived utility of gains and losses. Journal of Personality and Social Psychology, 55, 710-717.

Isen, A. M., \& Patrick, R. (1983). The effect of positive feelings on risk-taking: When the chips are down. Organizational Behavior and Human Performance, 31, 194-202.

Isen, A. M., Rosenzweig, A. S., \& Young, M. J. (1991). The influence of positive affect on clinical problem solving. Medical Decision Making, 11, 221-227.

Isen, A. M., Shalker, T., Clark, M., \& Karp, L. (1978). Affect, accessibility of material in memory, and behavior: A cognitive loop? Journal of Personality and Social Psychology, 36, 1-12.
Kahn, B. E., Greenleaf, E., Irwin, J. R., Isen, A. M., Levin, I. P., Luce, M. F., Pontes, M. C. F., Shanteau, J., Vanhuele, M., \& Young, M. J. (1997). Examining medical decision making from a marketing perspective. Marketing Letters, 8, 361-375.

Kahn, B. E., \& Isen, A. M. (1993). The influence of positive affect on variety-seeking among safe, enjoyable products. Journal of Consumer Research, 20, 257-270.

Kassirer, J. P. (1994). Incorporating patients' preferences into medical decisions. New England Journal of Medicine, 330, 1895-1896.

Kim, J., Allen, C. T., \& Kardes, F. R. (1996). An investigation of the mediational mechanisms underlying attitudinal conditioning. Journal of Marketing Research, 33, 318-328.

Kraiger, K., Billings, R. S., \& Isen, A. M. (1989). The influence of positive affective states on task perception s and satisfaction. Organizational Behavior and Human Decision Processes, 44, 12-25.

Kuder, J. M. (1995). The current environment for medical decision making: Alternative efficiency concepts and decision motivation. Motivation and Emotion, 19, 221-236.

Kuder, J. M., Isen, A. M., \& DeLia, D. (1996, June). Which elderly patients benefit from primary care office-based case management? Paper presented at the annual meeting of the Association for Health Services Research, Atlanta, GA.

Lee, A., \& Sternthal, B. (1999). The effects of positive mood on memory. Journal of Consumer Research, 26, 115-127.

Lewinsohn, S., \& Mano, H. (1993). Multiattribute choice and affect: The influence of naturally occurring and manipulated moods on choice processes. Journal of Behavioral Decision Making, 6, 33-51.

Mackie, D. M., \& Worth, L. T. (1989). Processing deficits and the mediation of positive affect in persuasion. Journal of Personality and Social Psychology, 57, 27-40.

Mackie, D. M., \& Worth, L. T. (1991). Feeling good but not thinking straight: The impact of positive mood on persuasion. In J. P. Forgas (Ed.), Emotion and social judgment (pp. 201-220). Oxford, England: Pergamon.

Mano, H. (1997). Affect and persuasion: The influence of pleasantness and arousal on attitude formation and message elaboration. Psychology \& Marketing, 14, 315-335.

Mednick, S. A. (1962). The associative basis of the creative process. Psychological Review, 69, 220-232.

Mednick, M. T., Mednick, S. A., \& Mednick, E. V. (1964). Incubation of creative performance and specific associative priming. Journal of Abnormal and Social Psychology, 69, 84-88.

Melton, R. J. (1995). The role of positive affect in syllogism performance. Personality \& Social Psychology Bulletin, 21, 788-794.

Murray, N., Sujan, H., Hirt, E. R., \& Sujan, M. (1990). The influence of mood on categorization: A cognitive flexibility interpretation. Journal of Personality and Social Psychology, 59, 411-425.

Nygren, T. E., Isen, A. M., Taylor, P. J., \& Dulin, J. (1996). The influence of positive affect on the decision rule in risk situations: Focus on outcome (and especially avoidance of loss) rather than probability. Organizational Behavior and Human Decision Processes, 66, 59-72.

O'Connor, A. M., Fiset, V., DeGrasse, C., Graham, I., Evans, W., Stacey, D., Laupacis, A., \& Tugwell, P. (1999). Decision aids for patients considering health care options: Evidence of efficacy and policy implications. Journal of the National Cancer Institute Monograph, 25, 67-80.

Pontes, M. C. F., \& Pontes, N. M. H. (1996). Variables that influence consumers' inferences about physician ability and physician accountability for adverse health outcomes. Health Care Management Review, 22(1).

Rucci, A. J., Kim, S. P., \& Quinn, R. T. (1998). The employee-customer-profit chain at Sears. Harvard Business Review, 76, 83-97.

Russell, J. A., \& Carroll, J. M. (1999a). On the bipolarity of positive and negative affect. Psychological Bulletin, 125(1), 3-30.

Russell, J. A., \& Carroll, J. M. (1999b). The Phoenix of bipolarity: Reply to Watson and Tellegen (1999). Psychological Bulletin, 125, 611-617. 
Schneider, B., Ashworth, S. D., Higgs, C., \& Carr, L. (1996). Design, validity, and use of strategically focused employee attitude surveys. Personnel Psychology, 49, 695-705.

Schneider, B., \& Bowen, D. E. (1985). Employee and customer perception s of service in banks: Replication and extension. Journal of Applied Psychology, 70, 423-433.

Schneider, B., White, S. S., \& Paul, M. C. (1998). Linking service climate and customer perceptions of service quality: Test of a causal model. Journal of Applied Psychology, 83, 150-163.

Schwarz, N. (1990). Feelings as information: Informational and motivational functions of affective states. In E. T. Higgins \& R. Sorrentino (Eds.), Handbook of motivation and cognition: Foundations of social behavior (Vol. 2, pp. 527-561). New York: Guilford.

Schwarz, N., \& Bless, H. (1991). Happy and mindless, but sad and smart? The impact of affective states on analytic reasoning. In J. P. Forgas (Ed.), Emotion and social judgment (pp. 55-71). Oxford, England: Pergamon.

Staw, B. M., \& Barsade, S. G. (1993). Affect and managerial performance: A test of the sadder-but-wiser vs. happier-and-smarter hypotheses. Administrative Science Quarterly, 38, 304-331.

Taylor, S. E., \& Aspinwall, L. G. (1996). Mediating and moderating processes in psychosocial stress: Appraisal, coping, resistance and vulnerability. In H. B. Kaplan (Ed.), Psychosocial stress: Perspectives on structure, theory, life-course, and methods (pp. 71-110). San Diego, CA: Academic.
Trope, Y., \& Neter, E. (1994). Reconciling competing motives in self-evaluation: The role of self-control in feedback seeking. Journal of Personality and Social Psychology, 66, 646-657.

Trope, Y., \& Pomerantz, E. M. (1998). Resolving conflicts among self-evaluative motives: Positive experiences as a resource for overcoming defensiveness. Motivation and Emotion, 22, 53-72.

Watson, D., Clark, L. A., \& Tellegen, A. (1988). Development and validation of brief measures of positive and negative affect: The PANAS scales. Journal of Personality and Social Psychology, 54, 1063-1070.

Watson, D., \& Tellegen, A. (1985). Toward a consensual structure of mood. Psychological Bulletin, 98, 219-235.

Watson, D., \& Tellegen, A. (1999). Issues in the dimensional structure of affect-effects of descriptors, measurement error, and response formats: Comment on Russell and Carroll. Psychological Bulletin, 125, 601-610.

Weiss, H., Nicholas, J. P., \& Daus, C. S. (1999). An examination of the joint effects of affective experiences and job beliefs on job satisfaction and variations in affective experiences over time. Organizational Behavior and Human Decision Processes, 78, 1-24.

Accepted by Frank Kardes. 